\title{
IDEACIÓN SUICIDA Y SINTOMATOLOGÍA OBSESIVO-COMPULSIVA EN UNA MUESTRA DE ADOLESCENTES
}

\author{
Francisco Serrano, Neus Barrantes, Edelmira Doménech, \\ Jordi E. Obiols y Susanna Subirá \\ Universidad Autónoma de Barcelona
}

\begin{abstract}
RESUMEN
El objetivo principal de este estudio es determinar la relación entre la sintomatología obsesivo-compulsiva (SOC) y la ideación suicida en una muestra elegida al azar $(n=1525)$ proveniente de la población de estudiantes de $8^{\circ}$ de EGB de la ciudad de Barcelona. Por otra parte, se estudiará la presencia de dicha sintomatología en la población origen de la muestra. La relación entre la alta SOC y la ideación suicida ha sido fuente de pocas investigaciones, las cuales frecuentemente se han centrado en el trastomo Obsesivo-compulsivo (TOC) y su relación con la conducta suicida. Es por ello que el estudio de la relación entre la alta SOC y ideación suicida no está exento de interés por el carácter novedoso del mismo. Los resultados indican que, tal y como se establecla en la hipótesis principal del trabajo, aquellos sujetos que cuyas puntuaciones son más elevadas en la escala que mide SOC presentan mayor ideación suicida. Se discute la posible implicación que dicho hallazgo supone en el marco de la práctica clínica y se establecen posibles estrategias para prevenir e identificar precozmente la conducta suicida en sujetos de alto riesgo.
\end{abstract}

Palabras clave: Alta sintomatología obsesivo-compulsiva, ideación suicida, sujetos de alto riesgo, detección precoz, implicaciones clinicas y epidemiológicas.

\section{ABSTRACT}

The main purpose of this study is to determine the relationship between obsessivecompulsive symptomatology and suicidal ideation in a large at random sample $(n=1525)$ drawn from the adolescent student population census of Barcelona. Another aim is to study the presence of obsessive-compulsive symptomatology (OCS) in that population. There is little information on the possible relationship between OCS and suicidal ideation, and many of the existing papers are centered on the Obsessive-Compulsive Disorder (OCD). Under such conditions, the existence of a possible relationship between OCS and suicidal ideation becomes a really interesting subject. Results show, as expected, that subjects with high OCS display more suicidal ideation than normal control subjects. Possible clinical implications of our findings and strategies for the precocious identificacion of suicidal behaviour in high risk children are discussed.

Key words: obsessive-compulsive symptomatology, suicidal ideation, high risk subjects, precocious identification, clinical and epidemiological implications. 


\section{INTRODUCCIÓN}

El objetivo principal de este estudio es determinar la relación entre la sintomatologia obsesivo-compulsiva (SOC) y la ideación suicida en una muestra elegida al azar $(n=1525)$ proveniente de la población de estudiantes de $8^{\circ}$ de EGB de la ciudad de Barcelona. Por otra parte, se estudiará la presencia de dicha sintomatología en la población origen de la muestra. Por último, se intentará estudiar la estrecha relación que se intuye entre la sintomatología obsesivo-compulsiva y la depresiva en dicha muestra.

Poca o nula es la información existente en torno a la posible relación entre la SOC y la ideación suicida, dado que la mayoría de estudios que se han llevado a cabo se centran fundamentalmente en el trastomo obsesivocompulsivo (TOC). Es por ello, que la presencia de un nexo entre la SOC y la ideación suicida adquiere un especial interés. Dada la existencia de un amplio consenso en cuanto a la aceptación de un vinculo relacional entre el TOC y la depresión (Goodwin, Guze y Robins, 1969; Gittelson, 1966; Videbech, 1975; Corryel, 1982; Rasmussen y Tsuang, 1982 y Vallejo y cols. 1988) y si consideramos la ideación suicida como uno de los síntomas básicos y patognomónicos del sindrome depresivo, no es difícil inferir la presencia de ideación así como conductas de carácter autolítico en sujetos con alta SOC. Por otra parte, se acepta generalmente que la presencia de alta SOC (y en mayor medida la presencia del TOC) se acompañan de sentimientos de caracter disruptivo y ego-distónico que pueden conducir con una alta frecuencia a ideación suicida y en ultima instancia a intentos autoliticos. A pesar de ello, algunos investigadores como Gittelson (1966) mantienen que los pacientes afectados de sindrome depresivo y en los cuales encontramos de forma concurrente SOC, llevan a cabo menos intentos autoliticos que aquellos que no presentan SOC. Corryel (1982) señala que, a pesar que la sintomatologia depresiva es común en pacientes con TOC, esta no siempre conduce a la aparición de ideación, amenazas o actos de caracter autolitico. Por su parte, Videbech (1975) niega el posible factor de protección que constituye la existencia de SOC en la conducta suicida, tal y como mantienen los dos autores citados con anterioridad. Zetin, en 1990 desarro-
Iló un modelo serotoninérgico del TOC. Dicho modelo ofrece un nexo de unión entre la ideación y la conducta suicida y la impulsividad, las compulsiones y la bulimia nerviosa. Recientemente, Oakeley-Brown (1991) llevó a cabo un extenso estudio epide-miológico de los trastornos de ansiedad. En dicho estudio, encontró una estrecha relación entre la conducta y la ideación suicida y las obsesiones y compulsiones en la población obsesivocompulsiva.

Otro vinculo entre dichas entidades psicopatológicas emerge de la investigación de la conducta suicida. En 1982, Rogers y cols. administraron la conocida escala de psicopatologia SCL-90-R a una muestra de 53 sujetos con alta ideación suicida. Sus resultados pusieron de manifiesto que dichos sujetos presentaban puntuaciones más altas en la subescala Obsesivo-Compulsivo que los sujetos pertenecientes al grupo control.

En conclusión, la relación entre la ideación, amenazas $\theta$ intentos suicidas y la SOC no aparece demasiado clara, a pesar de que los resultados fruto de estudios tanto farmacologicos como epidemiológicos parecen demostrar la existencia de un estrecho vínculo entre ambas entidades psicopatológicas. Etiológicamente, la SOC y la ideación suicida parecen compartir, en principio, una base biológica común.

El presente estudio intentará aportar datos que clarifiquen un tema tan confuso, estudiando la ideación suicida y la SOC en una amplia muestra de 1525 sujetos adolescentes, todos ellos estudiantes de $8^{\circ}$ de EGB de la ciudad de Barcelona. Nuestra hipótesis inicial supone que aquellos sujetos que presentan alta sintomatología obsesivo-compulsiva presentarán mayor ideación suicida que aquellos sujetos que presenten baja SOC.

\section{MÉTODO}

El presente trabajo de investigación se enmarca en un proyecto más amplio cuyo objetivo principal es profundizar en el estudio de la epidemiologia de la depresión y la ideación suicida en la población adolescente de Barcelona. Los individuos que componian la muestra eran estudiantes de $8^{\circ}$ de EGB de colegios públicos y privados. El procedimiento de recogida de datos se llevó a cabo en la ciudad de Barcelona durante los años 1992 y 1993. 
El intervalo de edad de los sujetos que componian la muestra oscilaba entre los 12 y los 16 años. Dado que el estudio se centraba en estudiantes de $8^{\circ}$ de EGB, la edad de la mayoria de sujetos estaba comprendida entre los 13 y los 14 .

El número de sujetos que deblan ser evaluados se obtuvo de una muestra al azar por el método de conglomerados de la ciudad de Barcelona. La unidad de aleatorización fue la escuela. Al inicio del estudio, el número total de adolescentes evaluados fue de 1525. Esta muestra es representativa de todos los estudiantes del mismo nivel escolar de la ciudad de Barcelona. La muestra se compone de $55 \%$ de niñas y $45 \%$ de niños, con una edad media de 13.44 anos. Una vez seleccionada la muestra, se administró a la misma el Children's Depressive Inventory (CDI, Kovacs, 1983) y el Leyton Obsessional Inventory (LOI-CV, Berg, 1986). En una segunda fase de dicho estudio epidemiológico, aquellos se entrevistó clínicamente mediante una escala semi-estructurada a aquellos sujetos que habian obtenido puntuaciones en el CDI por encima del punto de corte establecido para la misma. En el presente estudio se aportan únicamente datos provenientes de la primera fase.

Para evaluar la presencia de alta sintomatologia obsesiva se utilizó una versión reducida del Leyton Obsessional Inventory, Child Version (LOI-CV, Berg y Rapoport, 1986) elaborada por Berg en 1986 para estudios epidemiológicos. Dicha versión consta de 20 items que demostraron aceptables propiedades psicométricas. La puntuación total se obtiene sumando aquellos items que puntúan en sentido positivo, con el que se obtiene un índice global de obsesividad. Siguiendo a Berg y cols. (1988) se utilizo el valor 15 como punto de corte. La escala fue traducida por los autores y ha demostrado aceptables propiedades psicométricas (los resultados del estudio psicométrico de dicha escala, elaborado por Serrano, Domenech, Salamero, Barrantes y Obiols están sometidos a publicación).

La presencia de sintomatología depresiva se evaluó mediante el Children's Depression Inventory (Kovacs, 1983). Se ha estandarizado para individuos con edades comprendidas entre 8 y 16 años. La escala está compuesta por 27 items que incluyen sintomas propios de la depresión en áreas como la afectividad, la cognición y la conducta. Cada una de las 27 preguntas de las que consta la escala ofrece la posibilidad de elegir entre tres opciones de respuesta. El individuo debe elegir una de las tres opciones (oscilando así la puntuación entre 0-2 puntos) las cuales deben reflejar su estado de ánimo durante las dos últimas semanas; La puntuación total, en consecuencia, varía de 0 a 54. La traducción castellana de dicha escala ha sido utilizada en diversos estudios, los cuales han demostrado aceptables propiedades psicométricas, tal y como destacan Domenech y Polaino-Lorente, 1990 y Doménech, Canals y Fernández-Ballart, 1992. Se midió la presencia de ideación suicida siguiendo las propuestas meto-dológicas de Doménech, Canals y Fernández-Ballart (1992). Dichos autores utilizaron el item 9 del CDI para medir la presencia de ideación suicida. Las tres posibles respuestas a dicho item eran: "no pienso en matarme", "pienso en matarme pero no lo harla" y "quiero matarme".

\section{RESULTADOS}

Presencia de SOC en la población estudiantil adolescente de la ciudad de Barcelona

Del total de la muestra inicial $(n=1525), 128$ sujetos (65 chicos, 63 chicas, con una edad media de 13,52 años) obtuvieron una puntuación por encima del punto de corte establecido para el LOI-CV (puntuación=15). La puntuación media de estos sujetos en dicha escala fue de 17,70, con una desviación estándar de 0,99 . La media para el conjunto de la muestra fue de 10,04, con una desviación estándar de 3,20 . (ver Tabla 1). Por lo tanto, nos hemos encontrado con 128 sujetos $(8,4 \%$ del total de la muestra). que pre-sentan SOC clinicamente significativa. Si escogemos aquellos sujetos que obtienen alta SOC y baja sintomatologla depresiva (puntuación por debajo del decil 10 en la escala CDI), nos en-contramos con 36 sujetos $(2,4 \%$ del total de la muestra). Dichos sujetos presentan únicamente alta SOC y los podriamos denominar Obsesivos Puros. Nuestros resultados parecen estar de acuerdo con los datos de prevalencia del TOC en la población adolescente (valor que oscila entre el 1 y el $4 \%$ según diversos autores). Deberiamos destacar que nuestros sujetos son individuos con alta SOC y no pacientes con TOC. 
Tabla 1.- Descripción de la muestra inicial y la submuestra de alta SOC

\begin{tabular}{|l|lllll|}
\hline & $\begin{array}{l}\text { Número } \\
\text { De sujetos }\end{array}$ & Chicos & Chicas & $\begin{array}{l}\text { Media } \\
\text { LOI-CV }\end{array}$ & DT \\
\hline Muestra General & 1525 & 688 & 837 & 10,04 & 3,20 \\
Sujetos alta SOC & 128 & 65 & 63 & 17,70 & 0,99 \\
\hline
\end{tabular}

Siguiendo el curso de nuestras hipótesis, esperábamos encontrar una relación entre la SOC y la sintomatologia depresiva, relación que se intuye en base a los trabajos epidemiológicos y a los clínicos. En nuestro estudio, 44 sujetos del grupo de 128 (34,4\% de los mismos) obtuvieron puntuaciones por encima del punto de corte establecido por Domenech y Polaino-Lorente para la escala CDI. Podemos afirmar, por lo tanto, que el $34,4 \%$ de los 128 sujetos con alta SOC presentaban, en el momento de la recogida de datos, sintomatologia depresiva clinicamente significativa.

En cuanto a la variable sexo, no encontramos diferencias significativas en función del género en cuanto a la SOC se refiere, aunque se observa una cierta tendencia a que las chicas obtengan puntuaciones más altas (Media LOICV Chicos $=9,86$, Media Chicas=10,16).

\section{Ideación suicida y SOC}

Hemos encontrado una relación significativa entre la ideación suicida y la alta SOC en la población estudiantil adolescente de Barcelona. Las pruebas de Chi-cuadrado demuestran diferencias estadisticamente significativas en cuanto a la respuesta al item 9 (item del CDI que mide la presencia de ideación suicida) entre los dos grupos estudiados: el grupo control (sujetos cuya puntuación estaba por debajo del decil 10 en la escala LOI-CV) y el grupo de alta SOC (ver Tabla 2). Podemos afirmar, por lo tanto, que los sujetos con alta SOC presentan mayor ideación suicida que el grupo control. Hemos sumado los sujetos que puntuaban 2 y 3 en item 9 del CDI para facilitar la interpretación de los resultados (solamente encontramos dos individuos que obtuvieran una puntuación 3 en el item 9 del CDI).

Tabla 2.- Sujetos con alta y baja SOC y su relación con la ideación suicida

\begin{tabular}{|l|cc|}
\hline & Baja SOC & Alta SOC \\
\hline $\begin{array}{l}\text { Punt CDI } \\
\text { (item 9) }=0 \\
\begin{array}{l}\text { Punt CDI } \\
\text { (item 9) }=1+2\end{array}\end{array}$ & 9 & 83 \\
\hline Chi-cuadrado $=31,29$ (2), $P<0,0001$ \\
\hline
\end{tabular}

\section{Discusión}

Esta investigación constituye el primer intento, del que tengamos conocimiento hasta la fecha, de relacionar la ideación suicida con la sintomatología obsesiva en una amplia muestra de adolescentes estudiantes de $8^{\circ}$ de EGB de Barcelona. Estudiar la sintomatologia obsesiva en una muestra de la población adolescente constituye un reto, dado que las obsesiones y compulsiones suelen ser experimentadas como egodistónicas, absurdas y sin sentido lo cual produce una tendencia a mantener en secreto su existencia. Por otro lado, cabe destacar que el presente trabajo tiene como base el estudio 
de individuos con sintomatologia obsesiva, y que, en principio, todavia no han desarrollado un cuadro clínico completo. Es por ello que los resultados obtenidos adquieren mayor relevancia tanto en su interpretación estadistica como en lo que a la importancia clinica y preventiva se refiere. Creemos necesario incidir en este punto dada la presunta normalidad de los sujetos así como el hecho de que se trata de individuos en una etapa precoz de la adolescencia, lo cual confiere gran importancia a las diferencias halladas en una muestra representativa de la población estudiante de EGB de Barcelona en la cual no esperábamos que las mismas fuesen tan significativas.

El análisis de las hipótesis suele tener un sentido confirmatorio. En principio, y centrándonos en la hipotesis principal del presente trabajo de investigación, los datos indican la existencia de mayor ideación suicida en los sujetos que presentan alta sintomatología obsesiva frente a aquellos sujetos con puntuaciones bajas en la escala que detecta sintomatologia obsesiva. Dada la escasa bibliografia existente sobre el tema, y puesto que los pocos estudios publicados se centran en el TOC y no en sujetos con alta sintoma-tología obsesiva, este estudio no está exento de gran interés e importancia. No debemos olvidar que la ideación suicida es un punto clave en el continuum que supone el acto sui-cida. Así, el hecho de que individuos con alta sintomatología obsesiva presenten ideación suicida es ciertamente alarmante y deberia ser objeto de una profunda reflexión cuya finalidad principal sea poner en alerta a padres y maestros sobre las posibles consecuencias de dicha ideación buscando, con todo ello, elaborar un programa de prevención de la conducta suicida.

En cualquier caso, nuestros resultados están más de acuerdo con los resultados de Videbech y Oakeley-Browne que con autores como Coryell y Gittelson, y suponen la negación del posible factor de protección que suponia la sintomatología obsesiva y en concreto el TOC en la conducta suicida. Tal y como hemos planteado previamente, la asociación entre la sintomatologia obsesiva y la ideación suicida estaba legitimizada a priori puesto que es lógico pensar en la existencia de ideación suicida en aquellos sujetos que presentan alta sinto-matologia obsesiva. Dichos individuos se encuentran frente la intrusión inesperada de ideas, pensamientos e imágenes que sienten como ajenas y parasitarias, produciéndoles malestar y una intensa ansiedad. No debemos olvidar, sin embargo, que se trata de sujetos que no padecen un trastorno completo, aunque el contenido de los items incluidos en el LOI-CV induce a pensar que la presencia de alta sintomatologia obsesiva es, como mínimo, disruptiva y ansiogénica.

Desde el punto de vista epidemiológico, hemos encontrado 128 sujetos $(8,4$ del total de la muestra) que superan el punto de corte establecido para el LOI-CV, lo cual parece indicar que existe un alto número de sujetos provenientes de la población general que presentan sintomatologia obsesivo-compulsiva clínicamente significativa. Estos sujetos, siguiendo la alta validez predictiva del LOI-CV (ver Berg y cols. 1988) son individuos en una alta situación de riesgo para el desarrollo de un TOC, y no sujetos que presenten sintomatologia que en un futuro constituya parte de el trastorno de personalidad obsesivo-compulsivo. Por otra parte, dichos individuos presentan mayor psicopatologia, ya que el $34,4 \%$ de los mismos obtuvieron puntuaciones por encima del punto de corte establecido para el CDI (lo cual indica presencia de sintomatologla depresiva clínicamente significativa). Estos resultados parecen confirmar el esperado vínculo entre el sindrome obsesivo y el depresivo, todo ello teniendo en cuenta que se trata de sujetos con alta SOC y de no sujetos con TOC, en los cuales deberiamos esperar mayor sintoma-tología depresiva, dado el incremento de la gravedad sintomatológica en los sujetos con TOC. En cualquier caso, nuestros resultados parecen estar en consonancia con las recientes investigaciones (Flament y cols., 1988, Hollingsworth y cols., 1980, Rapoport, 1986 y Degonda y cols., 1993) que defienden un inicio infanto-juvenil de la patologia obsesiva, concretamente antes de los 20 años.

Un interés especial para la presente investigación supone la cantidad de sujetos obtenida (36 individuos) tras elegir aquellos sujetos con puntuaciones bajas en el CDI y altas en el LOI-CV. Esta cifra supone el $2,4 \%$ del total de individuos y concuerda con las cifras epidemiológicas que estiman la prevalencia del TOC entre un 1-4\%. Probablemente, estos sujetos sean aquellos con mayor riesgo de padecer un TOC en un futuro próximo con lo cual su lo- 
calización, seguimiento y terapéutica seria de suma utilidad, enmarcándonos así en una política de prevención.

Por otra parte, la variable sexo no parece ejercer influencia en la puntuación en el LOICV. Ello podría ser debido al procedimiento de recogida de datos (debemos señalar que intentábamos identificar sujetos con alta SOC, no sujetos con TOC).

La amplia muestra inicial de nuestro estudio confiere fiabilidad a los resultados de tal forma que se pueden extrapolar las conclusiones a las que se han llegado a la población adolescente de la cual proviene la muestra. Cabe remarcar que los sujetos han sido elegidos mediante un muestreo al azar realizado en la totalidad de la población escolarizada de Barcelona, lo cual confiere a la muestra una importante repre-sentatividad sociocultural. Por otra parte, el hecho de que la muestra esté compuesta por adolescentes probablemente da más significación a nuestros resultados, asi como el hecho de que se trata de una población normal en la cual no deja de ser sorprendente encontrar diferencias tan significativas.

Futuras investigaciones deberian centrarse en el estudio de la prevalencia de la ideación suicida en el seno del TOC más allá de la sintomatología obsesiva.

Por último, señalar que deberiamos considerar tanto el TOC como la presencia de alta SOC entidades psicopatológicas de alto riesgo dado que llevan consigo, de forma inherente, la posibilidad de desarrollar una conducta suicida. Tal y como señaló E. Hollander en el $1^{\text {st }}$ In-ternational Congress on Education and Progress in OCD, "el TOC debería ser entendido como una psicopatologia de alto riesgo en el sentido de que puede ser considerada como una amenaza para la vida"

\section{REFERENCIAS BIBLIOGRÁFICAS}

Berg, C. J., Rapoport, J.L. y Flament, M (1986). The Leyton Obsessional Inventory-Child Version. Joumal of the American Academy of Child and Adolescent Psychiatry. 25 (1), 84-91.

Berg. C. Z., Whitaker, A., Davies, M., Flament, M. y Rapoport, J.L (1988). The survey form of the leyton Obsessional Inventory-Child Version: norms from an epidemiological study. Journal of the American Academy of Child and Adolescent Psychiatry. 27 (6), 759-763.

Black, A. (1974). The natural history of obsessional neurosis. En: H. R Beech, Obsessional States. Londres: Methven.

Coryell, W., Noyes, R. y Clancy, J. (1982). Excess of mortality in panic disorders. A comparison with primary unipolar depression. Archives of General Psychiatry. 309, 701-703.

Degonda, M., Wyss, M. y Angst, J. (1993). The Zurich study. XVIII. Obsessive compulsive disorders and syndromes in the general population. European Archives of Psychiatry and Clinical Neurosciences. 243, 16-22.

Domènech, E., Canals, J. y Fernández-Ballart, J. (1992). Suicidal ideation among spanishschoolchildren: a three-year folow-up study of pubertal population. Personality and Individual Differences, 13 (9), 1055-1057.

Domènech, E. y Polaino-Lorente, A. (1990). Epidemiologia de la Depresión Infantil. BarceIona: Espaxs.

Flament, M.F, Whitaker, A., Rapoport, J.L. y cols. (1988). Obsessive compulsive disorder in adolescence: An epidemiological study. Journal of the American Academy of Child and Adolesc Psychiatry. 27: 764-771

Gittelson, N. (1966). The fate of obsessions in depressive psychosis. British Journal of Psychiatry. 112, 705-708.

Goodwin, D., Guze, S. B. y Robins, E. (1969). Followup studies in obsessional neurosis. Archives of General Psychiatry. 20, 182-187.

Hollingsworth, C., y cols. (1980). Long term outcome of obsessive-compulsive disorder in childhood. Joumal of the American Academy of Child and Adolescent Psychiatry . 9, 134-144.

Jenike, M. A. (1983). Obsessive-compulsive disorder. Comprehensive Psychiatry. 24 (2), 407 427.

Kovacs, M (1983) The Children's Depression Inventory: A Self Rated Scale For School-aged Youngsters. Manuscrito no publicado, University of Pittsburg.

Oakeley-Browne, M.A. (1991). The epidemiology of anxiety disorders. International Review of Psychiatry. 3 (2), 243-252.

Rasmussen, S. y Tsuang, M. (1986). Clinical characteristics and family history in DSM-III obsessive compulsive disorder. American Joumal of Psychiatry. 143, 317-322.

Rogers, J y cols. (1992). Help for families of suicide: Survivors Support Program. Canadian Journal of Psychiatry. 27 (6), 444-449. 
Serrano, F, y cols. Psychometric characteristics of the Leyton Obsessional Inventory-Child Version in a spanish adolescent population (en preparación).

Vallejo, J., Olivares, J., Marcos, T. y cols. (1988). Dexamethasone supression test and primary obsessional compulsive disorder. Comprehensive Psychiatry. 9, (5), 498-502.
Videbech, T. (1975). The psychopathology of anancastic endogenous depression. Acta Psychiatrica Scandinavica. 52, (3), 336-373.

Yaryura-Tobias, J. y Neziroglu, F (1983). Obsessivecompulsive disorders. Nueva York: Marcel Dekker.

Zetin, M. (1990). Obsessive-compulsive disorder. Stress-Medicine. 6 (4), 311-321. 